\title{
Using the Multisensory approach to teach students with Dyslexia
}

\author{
Khalid Mohammed Almahrag (Corresponding author) \\ Special Education, King Saud University \\ 3749 Riyadh 12372 - 7959, Kingdom of Saudi Arabia
}

Tel: 0966504440059 E-mail: almahrej@yahoo.com

\begin{abstract}
Dyslexia is a severe learning difficulty meeting many children in today's educational system. Meeting all of the individual needs of all children is difficult, as any educator today knows. That task is made even more complicated when the child has a diagnosed learning difficulty such as dyslexia. Unfortunately, it appears that there is no known medical care to alleviating the cognitive processing difficulties presented with dyslexia. Therefore, educators must use effective teaching strategies for children with dyslexia to help them become successful in an academic environment (Rupley \& Blair,1983). One of the primary adjustments that must be made is how dyslexic children are taught to read and write. Dyslexic children need a different approach to learning language from that employed in most classrooms. They need to be taught, slowly and ultimately, the essential elements of their language - the sounds and letters representing them - and how to put these together and take them apart. They have to use lots of practice in having their writing hands, eyes, ears, and voices working together for the aware organization and conservation of their learning. Current research converges on the efficacy of explicit, structured language teaching for children with dyslexia. Multisensory teaching is simultaneously visual, auditory, and kinesthetic-tactile to strengthen memory and learning. Therefore, being taught by a multisensory approach, children have the advantage of learning alphabetic patterns and words by utilizing all their senses. This paper will provide an overview of the problems faced by dyslexic children and how teachers using a multisensory approach can modify their instruction to accommodate these difficulties.
\end{abstract}

Keywords: Multisensory approach, Dyslexia, Teaching

DOI: 10.7176/JLLL/81-06

Publication date:September $30^{\text {th }} 2021$

\section{Introduction}

The terms "dyslexia" and "specific learning difficulties are often used synonymously, although some authorities would see "specific learning difficulties as an umbrella term for a range of learning difficulties, of which 'dyslexia' is one variant. At a very general level, educationalists and particularly educational psychologists tend to prefer the term "specific learning difficulties" and clinicians, voluntary organizations, and concerned laypeople the term "dyslexia. These differences of opinion can be seen partly due to the different perspectives that educationalists and clinicians are likely to have.

Although at present there is no one agreed definition of dyslexia, the following is still widely used. Dyslexia is a disorder manifested by difficulty in learning to read despite conventional instruction, adequate intelligence opportunities. It is dependent upon fundamental cognitive disabilities, which are frequently of constitutional origin.

Dyslexia affected males more than females between 3:1 and 5:1. The majority of experts agree that about $4 \%$ of the population is affected severely (Bright Solutions for Dyslexia, 2005). This figure is based on the rate of pupils who have received regular schooling and who do not have severe emotional, social or medical etiology, but whose literacy development by the end of the primary school is more than two years behind levels which would be supposed based on chronological age and intelligence. However, possibly as many as a further $6 \%$ of the population may be more mildly affected (e.g., spelling).

The biology of dyslexia has been investigated in various studies that have corroborated a difference in brain anatomy, organization, and acting. Research has also presented that the effects of dyslexia are due - at least, in part - to heritable weight (Bright Solutions for Dyslexia, 2005). The latest brain imaging methods and the encephalographic recording of the electrical function, and even post-mortem examination reveal a range of functional and structural cerebral anomalies of children with dyslexia.

Although it is a disability, dyslexia are not a disease, nor can it be healed. Indeed, the neurological distinctions found in dyslexia may confer advantages for some individuals (e.g., in visual or perceptual skills), which may to 
some extent clarify the evident paradox that some children who have problems with elementary skills such as reading, and writing can notwithstanding be exceptionally gifted in other areas.

\section{Research into a justification for a multisensory approach}

There is an increasing body of evidence supporting structured, multisensory teaching. Current research, much of it supported by the National Institute of Child Health and Human Development (NICHD), converges on the effectiveness of explicit, structured language teaching for children with Dyslexia (Riddell, Brown \& Duffield, 1995). A multisensory structured approach is commonly endorsed and practiced by teachers of children with a wide range of learning difficulties. Although clinicians and teachers have embraced multisensory teaching strategies since the earliest teaching guides were written, these strategies have seldom been well defined, and clinical wisdom has been waiting for scientific research ratification and explanation (Geschwind,1982). With current consensus findings concerning the nature of reading and spelling development, the efficacy of certain reading instruction practices, and the relationship between brain function and learning, we are closer to understanding why expert teachers have, for generations, been committed to structuring multisensory approaches.

To justify the significance of multisensory teaching, we will discuss how it has been implemented in the past, the instructional practices that are consistently supported by research, the role of the multisensory component in the effective instruction of language skills, some recent findings from neuroscientific studies, and what additional research might be needed to ratify the beliefs and practices of experienced clinicians who subscribe to these approaches.

Since the mid-1990s, several outstanding instructional programs have become organized under the description of multisensory structural education (Johnson \& Robertson,1999). They include techniques for linking eye, ear, voice, and hand in symbolic learning. The term multisensory is used generically to refer to any learning activity that comprises the use of two or more sensory modalities at the same time to take in or express information.

Farrell, Pickering, North, and Schavio (1995) investigated the use of multisensory strategies. In the initial phase of the study, nearly 30 senior clinicians who were directors of teacher education programs were asked in a survey to specify multisensory techniques included into each of the instructional objectives typical for lessons in their program cited by (Riddell, Brown \& Duffield,1995). Studies have shown that multisensory strategies can be used to improve phonological and decoding skills and improve spelling. For instance, Farrell refers to the OrtonGillingham teaching approach for students with dyslexia as a time-tested, research-based approach to teaching reading in a straightforward and multisensory way. The idea is simultaneous input involving all senses. Children are taught by having them look at a letter, say it, hear it and perhaps trace or write it simultaneously. This strengthens sound-symbol association and can significantly help those having problems in this area.

Multisensory instruction sends information along multiple pathways in the brain and accommodates a variety of learning styles. For example, students with dyslexia can write words in a sand tray or on a carpet square with two fingers while saying them out loud, clap syllables as they hear them during reading instruction, or act out action verbs and prepositions with body movements and props. All students find such multisensory instruction fun and motivating. Educational psychologists of the late 19th century promoted the theory that all senses, comprising the kinesthetic sense, are included in learning (Johnson \& Robertson,1999). This theory was presented through descriptions of typical children who used tracing to bolster visual memory. Steadfast with this theory were observations that the loss of acquired reading ability due to impaired visual memory in adults with brain effect could be bypassed through a kinesthetic modality (Johnson \& Robertson,1999).

The late 19th-century medical literature also discusses the use of "bypass" strategies in children who had lost their ability to read because of cerebral dysfunction (Johnson \& Robertson,1999). Hinshelwood was the first physician to advocate an unconventional instructional approach for written language disorders in children identified as "ord blind" (Department of Education and Science,1975). On the supposition that reading disability was due to underdevelopment or injury of the brain, he recommended instruction using an alphabetic method to request as many cerebral centers as possible.

S.T. Orton, a neurologist, was the first to write in the American medical literature on word blindness (Johnson \& Robertson,1999). He claimed a physiological failure was to develop a dominant language hemisphere to subsume reading, writing, and spelling. He appealed for education methods based on the simultaneous association of visual, auditory, and kinesthetic fields. For instance, by having a child sound the visually presented word and establish consistent directionality by following the letters with the fingers during sound synthesis of syllables and words.

Research has contributed an explanation of why phonics instruction is significant and influential for children 
learning to read and spell an alphabetic orthography (Riddell, Brown \& Duffield,1995). Skilled reading needs accurate processing of the internal details of words - their phonological, morphological, and orthographic features. Children must represent the linguistic structure of words they are learning to read in their minds, not only at the phoneme level but at other levels of language structure as well, particularly morphology or the meaningful parts of words. Although it appears that good readers guess at words or that they read whole words as units, good readers, in fact, the process virtually every letter of the words they read and are able, on-demand, to translate print to speech quickly and efficiently. The fluency of this translation process allows an excellent reader to attend to the meaning of what is read. Therefore, it is logical that effectual instruction with poor readers would enlarge their awareness of phonemes and other linguistic units. The speech-to-print translation process would become a focus of teaching until the children read fluently enough to concentrate on comprehension.

Indeed, numerous studies of Orton (1997) have shown that poor readers are marked by weaknesses in phoneme awareness, slow and incapable decoding skills, incorrect spelling, and connected language-processing difficulties Poor reader's problems are linguistic and are related both too inaccurate and too incompetent linguistic coding at elementary levels of word and subword processes (Bright Solutions for Dyslexia,2005). When readers cannot decode print precisely, comprehension is impaired; too much mental strength is being used to solve the message, and too little is available for making meaning. Effective instruction addresses these issues as entirely and methodically as possible. The structured, multisensory teaching is simultaneously visual, auditory, and kinesthetic-tactile to enhance memory and learning. Connection is consistently made between the visual, auditory, and kinesthetic-tactile pathways in learning to read and spell. Dyslexic children need a specific approach to learning a language that differs from that taught in most classrooms. They need to be introduced slowly and thoroughly. They have to have lots of practice in having their writing hands, eyes, ears, and voices working together for the conscious organization and retention of their learning (Riddell, Brown \& Duffield,1995).

Empiric support for the power of structured, multisensory techniques remains elusive in recent studies of reading instruction. Nevertheless, academic help for the added benefit of multisensory approaches can be sought from the cognitive and neurological sciences. One logical explanation might be founded on the design of memory itself.

an elementary reading specialist, Gayle Newman (2003) is constantly looking for reading error patterns, diagnosing reading strengths and weaknesses, and then searching for solutions to provide each person with dyslexia with successful reading experiences. It appeared that in his research, one group of children had internalized the familiar routine of rereading questions while the other group had not. He could not find an obvious explanation. While focusing on instruction, he recalled his educational background, which emphasized the importance of a multisensory approach for increasing the possibility of every child's success. He considered the fact that children who are struggling readers may need to be reached through many senses.

Research (The National Institutes for Health,1999) has supported the use of multiple modalities to teach children with dyslexia. For example, Thornton successfully used a multisensory approach to teach additional facts. The Multisensory Basic Fact Program (MBFP) intervention included a counting-on strategy; mnemonics for doubles, near doubles, and 10s; and visual, auditory, and kinesthetic instruction. Second through sixth graders who had previously been assigned to remedial math classes participated. Teachers used the MBFP for math instruction during two 20-min sessions per week. The rest of the week, the traditional math program was taught. Results showed that each grade level improved mean performance at the end of the 11-week instructional period.

\section{Using multisensory teaching to supporting support dyslexic children}

Most teaching in schools is done using either vision or hearing (auditory sensations). The child's eyesight is used to read the information, look at graphs or pictures, or read what is on the teacher's board. The child's sight may be affected by difficulties with tracking, visual processing, or seeing the words become unclear or move around (Cox,1983). The child's hearing may be sufficient on a hearing test, but auditory memory or auditory processing may be weak. The response involves using more of the child's senses, particularly the use of touch and movement. This will give the child's brain tangible and kinetic memories to hang on to and the visual and auditory ones.

Several activities are incorporating multisensory techniques can be used. One such activity that has received increasing attention in the literature is word sorts. Several words containing particular features are presented to children during a word sort, who then group the words according to specific phoneme-grapheme, morphological, or orthographic patterns. The rationale for this type of activity is that by paying close attention to the word's spelling and then classifying it into a group of similar words, the child is encouraged to form generalizations about spelling patterns that can then be used in learning new words. Such activities may bridge the gap between 
the number of words that can be explicit "aught" and the number of "aught" through text exposure. The flexibility of the approach has made it effective in standard education classrooms as well. Order of presentation and multi-modal techniques such as talking, seeing, and feeling are significant components to facilitate learning. Repetition, review, and drill attempt to make skills automatic. Frequently, programs will work with fundamental language skills after the traditional curriculum has moved away from these skills (Cox,1983).

Teachers who utilize this approach teach dyslexic children to link the sounds of the letters with the written symbol. Children also connect the sound and symbol with how it feels to form the letter or letters. As children learn a new note or pattern, they carefully track, copy, and write the letter(s) while saying the corresponding sound. The sound may be made by the teacher and the letter name(s) given by the child. Children then read and spell words, phrases, and sentences using these patterns. Teachers and their children rely on all three pathways for learning rather than concentrating on a "right-word" or memory method, a "racing method," or "phonetic method" alone (Clark, Diana, Uhry \& Kellogg,1995).

By presenting the letters simultaneously through hearing, seeing, and feeling, the children's weaknesses are facilitated by uniting their learning pathways. This multisensory approach ensures automatic memory, which is difficult for those who lack natural fluency in language learning. Progress is made by going from the elementary to the more difficult tasks, building in much reinforcement, and proceeding as fast as possible, but as slowly as necessary to master the essential elements. Appropriate words are used to clarify sounds. New concepts are practiced until they become mechanical responses. Careful packing structured but not programmed, methods, and a sequential presentation combines reading, writing, and spelling to help the dyslexia child succeed.

Each person with dyslexia is unique, but the multisensory approach is adaptable enough to serve a vast range of ages and learning differences. For example, The Hickey Multisensory Language Course is widely used throughout the U.K. by teachers working with dyslexic learners of all ages (Bright Solutions for Dyslexia,2005). The more severe the learning differences, the bigger the need for an individualized, multisensory, sequential approach. In the hands of a skilled, trained tutor, children who have not learned commonly show significant progress in a few months.

A multisensory approach can be helpful to many (Cox,1983). According to the Institute of Child Health and Human Development research, multisensory teaching has also proven effective with dyslexic children (Riddell, Brown \& Duffield,1995). It comprises direct instruction in the sounds of letters, how the sounds are made in the mouth and throat, and how letters are written. Phonics is taught in a logical, sequential, carefully arranged manner which begins with simple, one-letter/one-sound symbols and progresses through digraphs, diphthongs, six kinds of syllables, roots, and affixes (Johnson \& Robertson,1999).

The multisensory approach is cognitive. The child is taught to think through language problems when reading and spelling rather than guess. While the English language comprises words from many foreign languages, making it problematic to learn, it is about $85 \%$ predictable when one knows the principles and generalizations governing its use (Cox,1983). This cognitive approach gives most of the guesswork out of reading and spelling and helps dyslexic children to get mastery over language learning.

An example will make this plain. The majority of dyslexic children experience problems over the direction of " and. "They can both be perceived as a stick with a circle at its base. However, on which side does the ring sit? A teacher might give the child a tactile (touchy/feely) example of the letter by getting the child to draw the letter giant on the carpet. This will allow the child to use their arms, their sense of balance, and their whole body (Cox,1983).

Some teachers buy letters made out of sandpaper so that the children can run their fingers over the letter "e, giving them a sturdy tactile memory. However, another way to give a steadfast tactile memory of "e is to create the letter out of plasticine, playdough, or clay. A usually used "rick to remember the direction of "e and 'd' is to exhibit the child the word "ed" on a card. This word begins with "e and ends with so that if you design a bed over the letters, the good part of "e will be the head of the bed, and the good part of the "d' will be the foot. We can draw a child lying on the bed to finish the picture. This gives a steady visual memory for the child to use each time the letter has to be written.

The net result of these activities will be that a child has a visual memory from seeing the letter, an auditory memory from hearing the sound it makes, a tactile memory from writing the letter in cursive handwriting, in the air, and from touching the sandpaper letter, and a kinetic (body movement) memory from having drawn the letter big on the carpet. Quite a multisensory experience!

This tried and tested approach has been used successfully for a long time, and its success lies in the fact that the 
dyslexic child is not restricted to visual and auditory experiences but can make use of other areas of the brain is trying to create clear memories of letters, words, and numbers that are hard to remember.

\section{Discussion}

Special educational needs, including dyslexia, must be identified, assessed, and addressed, mainly within an inclusive mainstream classroom. Special programs such as Orton-Gillingham, Hickey Multisensory Language Programmed, Beat Dyslexia, and Multisensory Teaching System for Reading are implemented as part of an Individual Education Plan. They need to be linked to the Literacy Hour. These programs have become a classic. They are widely used throughout the U.K. by teachers working with dyslexic learners of all ages and are a core text for university courses directed at specialist teachers. Moreover, they have influenced teaching literacy skills, including the National Literacy Strategy Framework for teaching. Children now have an entitlement to access to the National Curriculum.

Orton-Gillingham's structured, multisensory approach was developed in the early 1930s by Anna Gillingham and master teachers (Bright Solutions for Dyslexia, 2005). Perhaps the most significant thing about the OrtonGillingham approach is that it is the first multisensory structured language approach developed for reading, spelling, and writing instruction for dyslexic children. The philosophy and principles of the O-G approach became the basis of so many other approaches developed later (Clay,1979). Orton, a physician, suggested that children who could not learn to read easily with different instructional approaches need a multisensory approach that uses information from the visual, auditory, and kinesthetic pathways of learning

The O-G structured, multisensory approach is the direct teaching of phonics. A teacher begins working with a child by introducing the most common consonants and vowels, one or two at a time. As each letter is presented, it is taught in a multisensory manner, so the child links how the letter looks with how it sounds and how it feels to form the letter. Once the basic consonants and vowels are mastered, they pass on to consonant blends and digraphs. Children also learn the English language rules, syllable patterns, and how to use roots, prefixes, and suffixes to study words (Cox,1983).

Hickey Multisensory Language Programmed is widely used in Special Education classes in schools. Depending on the child's specific areas of deficiency, the Hickey method should be used selectively. In the Hickey Multisensory Language Programmed, the child commences with letters, letter blends, analysis of mixtures, phonic analysis of common words, polysyllabic words, and syllable division. Hickey, the originator of the Hickey Multisensory Language Programmed, asserts that difficulty in reading and writing relates directly to defects of sensual perception. Children affected by such defects are often unable to learn in the usual manner. They must be taught to reinforce other sensory modalities such as visual, auditory, kinesthetic, and tactile.

Hickey's objectives are that the child finds some way to write words, be motivated to read these words and extend his reading to other materials. Typed printing is recommended for these exercises. Each child should be given reading prescriptions that benefit him most. Tracing words are employed by this method-the child says the letter or word while tracing it with his finger. Lexicography (collecting words) is also used. The words are placed in an alphabetical file for later reidentification, are written as whole units, and not copied part by part. Children must be taught the meaning of each new word learned. Picture clues are placed on the back of the cards as a reward after the word has been read correctly by the child. The person with dyslexia writes the word, naming each letter as they write. This step translates the sound into a written equivalent and as a motor, tactile or kinesthetic program. Naming each letter helps to map out the correspondences.

Beat Dyslexia - series of activities with graded phonics program (Bright Solutions for Dyslexia, 2005), Beat Dyslexia is recommended for homeschooling parents, A step-by-step multisensory literacy program for all Dyslexic Pupils. It includes six-packs, which cover changing Letters \& sounds. The program is structured so that all goals are small \& reachable. At the beginning of each session, children have to make an ark of the alphabet and then have to take out and feel the shape of the letter being covered in the session. They then continue to say the sound the letter makes. Following this, children have to design the letter in the air as big as they can. They also draw the note on the back of each other's hands to feel how the letter is written. The next step is to write the letter on paper, first tracing over the note and then writing a sequence. After that, children have to listen to a tape and markdown in the accurate box where the letter sound comes in the word. After this, children have talks with the teacher or parents, and if they have got any problems, they can listen to the tape a second time and make any corrections. Beat Dyslexia is an in-house spelling program with an integrated system for handwriting, spelling, and reading. The technology combines a wide range of laptops, P.C.s, multimedia systems, spellcasters, Dictaphones, tape recorders, a video presenter (Bright Solutions for Dyslexia, 2005).

Multisensory Teaching System for Reading (MTSR) - this teaching pack has been produced as the result of 
cooperation between the Manchester Metropolitan University and the British Dyslexia Association (BDA) (Johnson \& Robertson,1999). Multisensory Teaching System for Reading (MTSR) is a well-evaluated program. The program helps children promote phonological awareness, ensures overlearning, and provides time for review and mastery. It is based on combined, structured, sequential multisensory delivery with frequent small steps. Johnson, Philips, and Peer (1999) researched the program's usefulness and found it encourages individual learning and helps children better their self-esteem.

Similar to Beat Dyslexia, Multisensory Teaching System for Reading is helpful for homeschooling parents because it is scripted. It is one of the wave three materials recommended by DFES for use in school by classroom assistants with small children. This has been successfully used in several states in the U.S. as their entire reading teaching curriculum. Teaching System for Reading is a scripted, multisensory program for teaching word-level reading, created for groups of children for 20 minutes a day, four days a week, over about 12-20 weeks (Johnson \& Robertson,1999).

Once children understand the phonological, morphological, and orthographic patterns, they will likely need many opportunities to practice and internalize the relationships. At this point, they can be given instructional strategies to help them memorize the spellings of particular words. Multisensory techniques, such as Gillingham and Stillman's(1997) Simultaneous Oral Spelling and Alpha to Omega, the Hickey, as well as computer programs, can help.

\section{Conclusion}

Children with dyslexia often show weaknesses in auditory and visual processing. They may exhibit weak phonemic awareness, meaning they are unaware of the role sounds play in words. Dyslexic children have difficulty rhyming words, blending sounds to make words, or segmenting words into sounds. They may also have problems acquiring a sight vocabulary. That is, dyslexic children do not learn the sight words supposed in the primary grades. As usual, they do not pick up the alphabetic code or system.

With the help of a multisensory approach, dyslexic children have the advantage of learning alphabetic patterns and words by using many senses. Young children in structured, sequential, multisensory intervention programs, also practiced in phonemic awareness, made practical decoding skills. Dyslexic children in clinical settings showed similar results for a wide range of ages and abilities.

The teachers use as many senses as possible to facilitate the pupils' ways of practicing and learning. In this way, strong learning channels are used, and they help build up stronger and essential ones. The work is structured. The Dyslexia Institute's Literacy Programmed introduces letters and groups of letters, strategies for mustering long words, "Ricky" words and spelling rules, indefinite order (Clark, Diana, Uhry \& Kellogg,1995). At each stage, the dyslexic child is working only with the part that has been covered during lessons. This creates confidence to muster longer words and passages. It also includes some effective routines for tackling work. The work is cumulative. Each part of the program leads on to the next, and the child can be assured that he is only expected to do work for which he has been well prepared. Multifarious and exciting activities are used for essential practice. Pupils must have systematic revision and "overlearn" until they automatically use sounds, letters, and rules in reading and writing (Stoane, Franks \& Nicholson,1993).

Teachers and parent like to use multisensory approaches because it is easy to use and also structured. It is adequate to use in general education classrooms and for one-to-one or small groups (Bright Solutions for Dyslexia,2005). The Hickey Multisensory Language Course is implemented as part of an Individual Education Plan linked to the Literacy Hour. Multisensory approaches are not limited to a set curriculum for a specific age group. Learning disabled, English as a Second Language, and regular education students have success using multisensory approaches. We think multisensory techniques beneficial strategies that children with dyslexia can learn from it.

\section{References}

Bright Solutions for Dyslexia. [Online]. Available from $:<$ http://www.dys- add.com/teach.html $>$ [Accessed 29 June 2020].

Clay, M.M. (1979). The Early Detection of Reading Difficulties, London, Heinemann.

Cox, A. R. (1983). Programming for teachers of dyslexics. Annals of Dyslexia, 33(1), 221-233

Clark, Diana Brewster, and Uhry, Joanna Kellogg, (1995). Dyslexia: Theory \& Practice of Remedial Instruction, Second Edition, Baltimore, MD, York Press.

Department of Education and Science (DES), (1975). Special Educational Needs (Warnock Report), 
London, HMSO Guide to Best Practices for Educators.

Geschwind, N. (1982). Orton was right, Annals of Dyslexia, 32,13-30.

Johnson, M., and Robertson, J. (1999). Hemispheric Stimulation by Computer for pupils with Dyslexia, Journal of Learning Disabilities. (Submitted)

Riddell, S., Brown, S., and Duffield, J. (1995). The Ethics of Policy-focused research in Special Educational Need in Clough, P., and Barton, L. Making Difficulties: Research and the construction of SEN, London, Paul Chapman Publishing.

Rupley, W.H., and Blair, T.R. (1983). Reading Diagnosis and Direct Instruction: a guide for the classroom. London: Houghton Mifflin

Stone, C., Franks, E. and Nicholson, J. (1993). Beat Dyslexia (Bk. 1-4), Wisbech, LDA

Wilson, J. (1994). Phonological Awareness Training, London, Educational Supply Publishers. 